\title{
miR-29c is downregulated in the ectopic endometrium and exerts its effects on endometrial cell proliferation, apoptosis and invasion by targeting c-Jun
}

\author{
MEI LONG ${ }^{1 *}$, XIAOHUI WAN ${ }^{2 *}$, XIAOLIN LA ${ }^{1}$, XIAOYUN GONG ${ }^{1}$ and XIA CAI ${ }^{1}$ \\ ${ }^{1}$ Productive Center, ${ }^{2}$ Department of Gynaecology, The First Affiliated Hospital \\ of Xinjiang Medical University, Urumqi, Xinjiang Uygur Autonomous Region 830054, P.R. China
}

Received June 24, 2014; Accepted January 16, 2015

DOI: $10.3892 / \mathrm{ijmm} .2015 .2082$

\begin{abstract}
Endometriosis is a prevalent and complex gynecological disease which affects $10 \%$ of women of reproductive age. Certain studies have suggested that a substantial number of microRNAs (miRNAs or miRs) are aberrantly or differentially expressed in the ectopic endometrium. To date, to the best of our knowlewdge, there is no report available on the role of miR-29 in the endometrium. In this study, we investigated the expression of the miR-29 family in the endometrium samples from women without endometriosis, as well as in paired ectopic and eutopic endometrium samples by reverse transcription-quantitative polymerase chain reaction (RT-qPCR). The results revealed that miR-29c was differentially expressed in the paired eutopic and ectopic endometrium samples. In addition, c-Jun was differentially expressed in the ectopic and eutopic endometrial tissues as determined by western blot analysis. Furthermore, the role of miR-29c in endometrial cell proliferation, invasion and apoptosis was examined in vitro. The results revealed that miR-29c exerted its effects on endometrial cells by suppressing cell proliferation and invasion, as well as promoting cell apoptosis. Furthermore, it was found that c-Jun was a novel target of miR-29c, and c-Jun reversed the effects of miR-29c on the proliferation, invasion and apoptosis of endometrial cells. To the best of our knowledge, this study is the first to identify miR-29c as a suppressor of endometriosis. Taken together, our results suggest that miR-29c exerts its effects on endometrial cell proliferation, apoptosis and invasion by inhibiting the expression of c-Jun. Our data
\end{abstract}

Correspondence to: Professor Xia Cai, Productive Center, The First Affiliated Hospital of Xinjiang Medical University, 137 Liyushan Road, Urumqi, Xinjiang Uygur Autonomous Region 830054, P.R. China

E-mail: caixia5512@163.com

${ }^{*}$ Contributed equally

Key words: miR-29c, endometriosis, c-Jun may provide a novel potential therapeutic target for the treatment of endometriosis.

\section{Introduction}

Endometriosis is a prevalent and complex gynecological disease which affects $10 \%$ of women of reproductive age (1), and is characterized by the growth of endometrial glandular epithelial and stromal cells outside the uterine cavity. Women of reproductive age with endometriosis often suffer from painful menstrual periods, chronic pelvic pain and infertility. This disease is multifactorial and factors, such as hormones, immune response and the environment may contribute to the susceptibility and progression of endometriosis (2-4). In the majority of cases, the spread of extrauterine endometrial tissue is due to retrograde menstruation and lymphatic dissemination (5).

microRNAs (miRNAs or miRs) are small non-coding RNAs, which are now known to play essential roles in the regulation of gene expression for cell proliferation and apoptosis, cell cycle, immune response and inflammation $(6,7)$. The deregulation and aberrant expression of these molecules has been implicated in a variety of human diseases, including cancer $(8,9)$.

Endometriosis displays a benign morphology; however, endometriotic cells have an invasive potential which is characterized by malignant cells (10). To date, to the best of our knowledge, there is no report available on the role of miR-29 in the endometrium. In the present study, we investigated the expression of miR-29a, miR-29b, miR-29c in endometrium samples from women without endometriosis, as well as in paired ectopic and eutopic endometrium samples. Furthermore, the role of miR-29c in endometrial cell proliferation, invasion and apoptosis was examined in vitro, and a novel target through which miR-29c exerts its effects on endometrial cells was identified.

\section{Materials and methods}

Tissue samples. The procedures for the collection of the tissue samples were approved by the Ethics Committee of the First Affiliated Hospital of Xinjiang Medical University, Urumqi, 
China. All patients signed an informed consent form in compliance with the code of ethics of the World Medical Association (Declaration of Helsinki). Paired eutopic and ectopic endometrium samples were collected from 20 women with endometriosis who underwent laparoscopic surgical procedures at the First Affiliated Hospital of Xinjiang Medical University. The patients were aged 25 to 42 years, had regular menstrual cycles and had not received hormonal therapy for at least 3 months prior to surgery. A total of 10 samples of endometrium from women with hysteromyoma were used as the controls. The tissues were from the proliferative phase of the menstrual cycle. After collection, the tissue samples were immediately frozen and kept in liquid nitrogen for further analysis.

Cell culture and transfection. The CRL-7566 endometriosis cell line was purchased from the American Type Culture Collection (ATCC; Manassas, VA, USA). This cell line was established from a benign ovarian cyst obtained from a patient with endometriosis. The cells were isolated from both the inner and outer surfaces of the cyst. The cells were cultured in Dulbecco's modified Eagle's medium (Invitrogen, Carlsbad, CA, USA) containing $10 \%$ fetal bovine serum (FBS; Gibco, Carlsbad, CA, USA). The cells were grown at $37^{\circ} \mathrm{C}$ in $5 \% \mathrm{CO}_{2}$. The miR-29c mimic, miR-29c inhibitor and negative control (NC), mutant and wild-type c-Jun 3'UTR, as well as the c-Jun overexpression plasmid and the shRNA plasmid were transfected into the cells using Lipofectamine 2000 reagent (Invitrogen) following the manufacturer's instructions and incubated at $37^{\circ} \mathrm{C}$ for $24 \mathrm{~h}$.

Luciferase assay. The targets of miR-29c were predicted by miRanda (http://www.microrna.org/) and c-Jun was identified to be a target of miR-29c. The sequences of human wild-type c-Jun 3' untranslated region (3'UTR) and mutant c-Jun 3'UTR were then cloned into the pGL3-control luciferase reporter vector (Promega, Madison, WI, USA). c-Jun 3'UTR reporter plasmids were co-transfected with the miR-29c mimic or negative control (NC) into the cells. pRL Renilla luciferase reporter vector was used as the internal control in each assay. Following transfection, cell lysates were collected and luciferase activity was measured using the Dual-Luciferase Reporter assay system (Promega).

MTT assay. MTT assay was used to evaluate cell proliferation. The cells were seeded into 96-well culture plates at a density of $1 \times 10^{4}$ cells/well. Following transfection, $10 \mu \mathrm{l}$ MTT solution $(0.5 \mathrm{mg} / \mathrm{ml}$; Beyotime, Shanghai, China) were added to each well followed by incubation for $4 \mathrm{~h}$. After dissolving the MTT formazan with DMSO (Sigma, St. Louis, MO, USA), cell proliferation was determined by reading the plates at $570 \mathrm{~nm}$ on a Multiskan Ascent 354 microplate reader (Thermo Labsystems, Waltham, MA, USA).

Cell invasion assay. Cell invasion assay was performed using Matrigel-coated Transwell plates (Corning, New York, NY, USA). Cells in serum-free medium at a final density of $5 \times 10^{4}$ cells $/ \mathrm{ml}$ were added to the upper chambers and incubated at $37^{\circ} \mathrm{C}$ for $12 \mathrm{~h}$, and cell medium containing $10 \% \mathrm{FBS}$ was added to the lower chambers. Following incubation, non-invaded cells were removed using cotton swabs, and the invaded cells were fixed in $95 \%$ ethanol and stained with hematoxylin. The number of invaded cells was determined by counting the stained cells under an inverted microscope (TS100; Nikon, Tokyo, Japan).

Flow cytometry (FCM). Cell apoptosis was assessed by FCM. After washing with phosphate-buffered saline (PBS), the cells were resuspended in binding buffer and stained with Annexin V and propidium iodide (PI) (Kaiji Biological Inc., Nanjing, China) following the manufacturer's instructions. Positive cells were detected and quantified by FCM (BD FACSAria; BD Biosciences, Franklin Lakes, NJ, USA).

Reverse transcription-quantitative polymerase chain reaction (RT-qPCR). Total RNA was isolated using TRIzol reagent (Invitrogen) according to the manufacturer's instructions. The miRNAs were isolated using a miRNeasy Mini kit (Qiagen, Hilden, Germany). Reverse transcription was performed using a RevertAid $^{\mathrm{TM}}$ First Strand cDNA Synthesis kit (Fermentas, Vilnius, Lithuania) and quantitative (real-time) PCR was carried out on a 7900 Real-Time PCR System using a SYBRGreen PCR kit (both from Applied Biosystems, Foster City, CA, USA) following the manufacturer's instructions. The results were analyzed using the comparative method following normalization of the expression values to U6.

Western blot analysis. Protein samples were extracted using a total protein extraction kit (Kaiji Biological, Inc.) and the protein concentration was determined using the BCA kit (Beyotime). The samples were separated in 10\% SDS-PAGE and transferred onto polyvinylidene fluoride membranes (Millipore, Billerica, MA, USA). The membranes were blocked in $5 \%$ non-fat milk overnight at $4^{\circ} \mathrm{C}$. After washing with PBS, the membranes were incubated with primary antibody [mouse monoclonal to c-Jun (ab119944), mouse monoclonal to $\beta$-actin (ab8226); Abcam, Cambridge, MA, USA] overnight at $4^{\circ} \mathrm{C}$, followed by incubation with horseradish peroxidase-conjugated secondary antibody (BA1050; Boster, Wuhan, Hubei, China) for $1 \mathrm{~h}$ at room temperature. The ECL detection kit (Pierce, Rockford, IL, USA) was used to detect the proteins.

Statistical analysis. Data were presented as the means \pm standard deviation (SD). The two-tailed Student's t-test was used to statistically analyze data between 2 groups. A P-value $<0.05$ was considered to indicate a statistically significant difference.

\section{Results}

Differential expression of miR-29 family in tissue samples. The differential expression of miR-29a, miR-29b, miR-29c in the endometrium samples from women without endometriosis, as well as in the eutopic and ectopic endometrium samples was determined by RT-qPCR. The results revealed that the expression of miR-29c progressively decreased from the samples of endometriosis to the those of the eutopic and ectopic endometrium, and the expression of miR-29c between the eutopic and ectopic endometrium was differed significantly $(\mathrm{P}<0.01)$. However, the expression levels of miR-29a and miR-29b did not differ significantly between the 3 groups (Fig. 1).

Differential expression of c-Jun in tissue samples. The expression of c-Jun in the endometrium samples from women without 

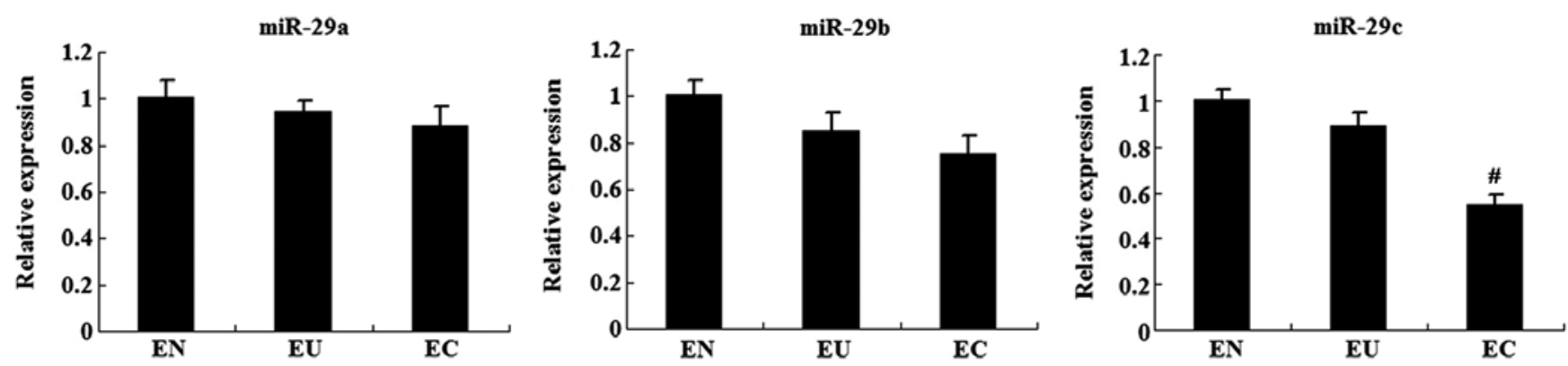

Figure 1. Differential expression of the miR-29 family in endometrial tissues determined by RT-qPCR. ${ }^{~} \mathrm{P}<0.01$ compared with the EU group. EN, samples from women without endometriosis; EU, eutopic endometrium; EC, ectopic endometrium.
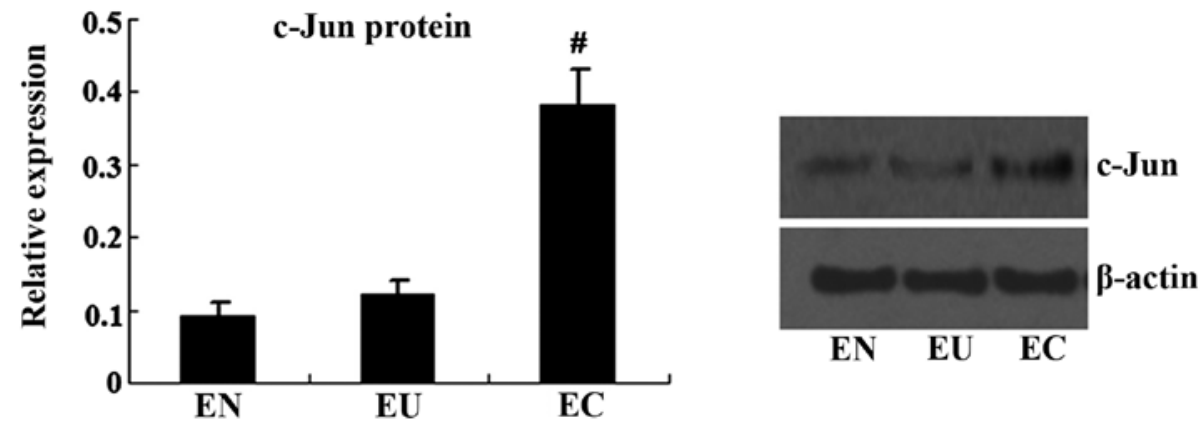

Figure 2. Differential expression of c-Jun in tissues determined by western blot analysis. " $\mathrm{P}<0.01$ compared with the EU group. EN, samples from women without endometriosis; EU, eutopic endometrium; EC, ectopic endometrium.

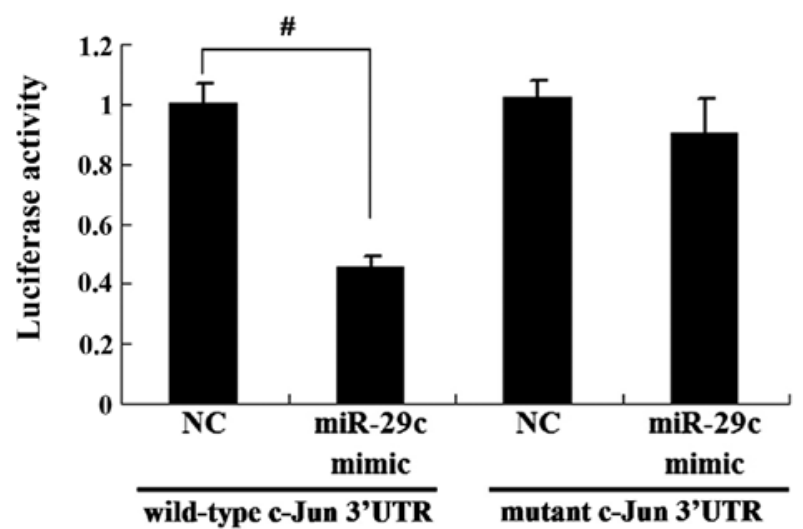

Figure 3. c-Jun is a direct target of miR-29c as determined by luciferase reporter assay. ${ }^{*} \mathrm{P}<0.01 . \mathrm{NC}$, negative control.

endometriosis, as well as in the eutopic and ectopic endometrium samples was examined by western blot analysis. There was no statistically significant difference in c-Jun expression between the group without endometriosis and the eutopic group (Fig. 2). However, the protein expression level of c-Jun was significantly higher in the ectopic group compared with the eutopic group $(\mathrm{P}<0.01)$.

c-Jun is directly regulated by $\mathrm{miR}-29 c$. In order to determine whether c-Jun is a direct target of miR-29c, wild-type c-Jun3'UTR or mutant c-Jun-3'UTR were co-transfected with the miR-29c mimic or NC. Luciferase assay revealed that transfection with the miR-29c mimic significantly inhibited the luciferase activity of wild-type c-Jun-3'UTR $(\mathrm{P}<0.01)$; however, the lucif- erase activity of mutant c-Jun-3'UTR did not differ statistically following transfection with the miR-29c mimic or NC (Fig. 3).

Furthermore, the effect of miR-29c on c-Jun expression was examined by western blot analysis. The results from RT-qPCR revealed that miR-29c expression was significantly upregulated in the miR-29c mimic-transfected group and downregulated in the miR-29c inhibitor-transfected group (Fig. 4A). Western blot results revealed a significantly decreased expression of c-Jun in the cells transfected with the miR-29c mimic; however, the expression of c-Jun was increased in the cells transfected with the miR-29c inhibitor (Fig. 4B).

Expression of miR-29c and c-Jun following transfection with the c-Jun overexpression or shRNA plasmid. The open reading frame (ORF) clone of homo c-Jun was subcloned into the pcDNA3.1 vector to induce the overexpression of c-Jun. We suppressed c-Jun expression using a specific shRNA plasmid. Transfection with the c-Jun overexpression plasmid did not alter the increase in miR-29c expression in the miR-29c mimic-tansfected cells (Fig. 5A). In addition, the c-Jun shRNA plasmid did not alter the decrease in miR-29c expression in miR-29c inhibitor-tansfected cells (Fig. 5B).

Cells transfected with the miR-29c mimic and c-Jun overexpression plasmid showed a significantly increased protein expression of c-Jun compared with the cells transfected with the miR-29c mimic only (Fig. 6A). However, transfection with c-Jun shRNA significantly downregulated the expression of c-Jun in the cells transfected with the miR-29c inhibitor (Fig. 6B).

$c$-Jun reverses the miR-29c-induced suppression of cell proliferation and invasion. In order to examine the effects of 

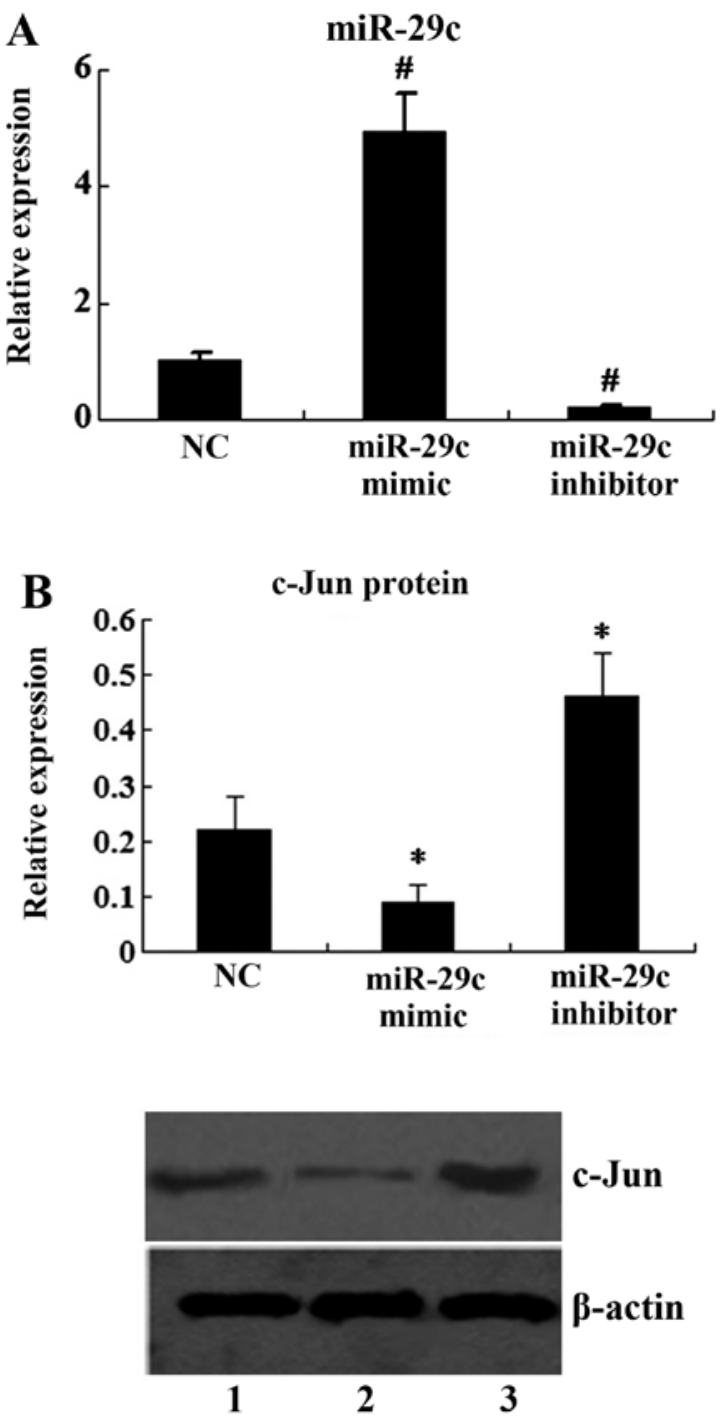

Figure 4. c-Jun expression is regulated by miR-29c. (A) mRNA expression of miR-29c following transfection with the miR-29c mimic or the miR-29c inhibitor in CRL-7566 cells. (B) Protein expression of c-Jun following transfection with the miR-29c mimic or the miR-29c inhibitor in CRL-7566 cells. Lane 1, NC; lane 2, miR-29c mimic; lane 3, miR-29c inhibitor. " $\mathrm{P}<0.05$ and ${ }^{\#} \mathrm{P}<0.01$ compared with the $\mathrm{NC}$ group. $\mathrm{NC}$, negative control.

miR-29c on cell proliferation, the miR-29c mimic or inhibitor were transfected into the cells, and MTT assay was used to analyze cell proliferation. Compared with the NC-transfected cells, the miR-29c mimic-transfected cells proliferated at a significantly lower rate (Fig. 7A). By contrast, transfection with the miR-29c inhibitor promoted cell growth (Fig. 7B).

Furthermore, the overexpression of c-Jun reversed the inhibitory effects of the miR-29c mimic on cell proliferation (Fig. 7A). Consistent with these results, transfection with c-Jun shRNA reversed the inductive effects of the miR-29c inhibitor on cell proliferation (Fig. 7B).

Cell invasion ability was determined by Transwell-Matrigel assay. Transfection with the miR-29c mimic resulted in significantly decreased cell invasion in comparison to transfection with $\mathrm{NC}$; however this effect was reversed by transfection with the c-Jun overexpression plasmid (Fig. 8A). As expected, transfection with the miR-29c inhibitor significantly increased
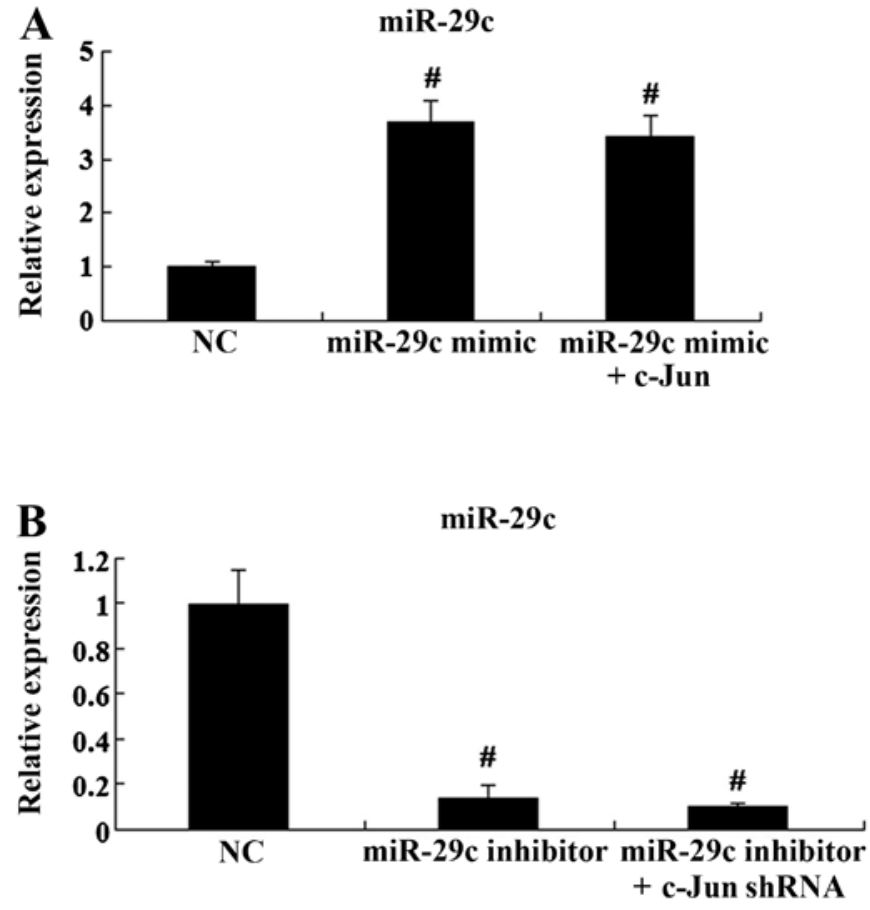

Figure 5. Expression of miR-29c mRNA following transfection with the miR-29c mimic/inhibitor and c-Jun overexpression/shRNA plasmid in CRL-7566 cells. (A) c-Jun overexpression plasmid did not alter the increase in miR-29c expression in miR-29c mimic-tansfected cells. (B) c-Jun shRNA plasmid did not alter the decrease in miR-29c expression in miR-29c inhibitortansfected cells. ${ }^{\text {P }}<0.01$ compared with the NC group. NC, negative control.

cell invasion ability, which was reversed by transfection with c-Jun shRNA (Fig. 8B).

c-Jun reverses the miR-29c-mediated induction of cell apoptosis. FCM was used to examine the cell apoptotic rates. The results revealed that compared with the cells transfected with $\mathrm{NC}$, the miR-29c mimic-transfected cells showed an increased cell apoptotic rate, while transfection with miR-29c inhibitor had the opposite effect. The overexpression of c-Jun reversed the increase in the cell apoptotic rate induced by the transfection with the miR-29c mimic. Consistent with these results, transfection with c-Jun shRNA reversed the inhibitory effects of the miR-29c inhibitor on cell apoptosis (Fig. 9).

\section{Discussion}

Pan et al (11) provided the first evidence of the expression of a unique set of miRNAs in the endometrium and endometrial cells, suggesting that a substantial number of miRNAs are aberrantly or differentially expressed in the ectopic endometrium. Ohlsson Teague et al (12) profiled miRNAs in paired samples of eutopic and peritoneal ectopic endometrial tissue from patients with endometriosis using miRNA microarray analysis. Of the miRNAs evaluated, 22 miRNAs showed a differential expression in the paired eutopic vs. the ectopic endometrium samples. Both the studies of Pan et al (11) and Ohlsson Teague et al (12) revealed that miR-29c was differentially expressed in the eutopic and ectopic endometrium. miR-29c belongs to the miR-29 family, and it has been suggested to be downregulated in certain types of cancer (13-16). In the present study, the expression levels 

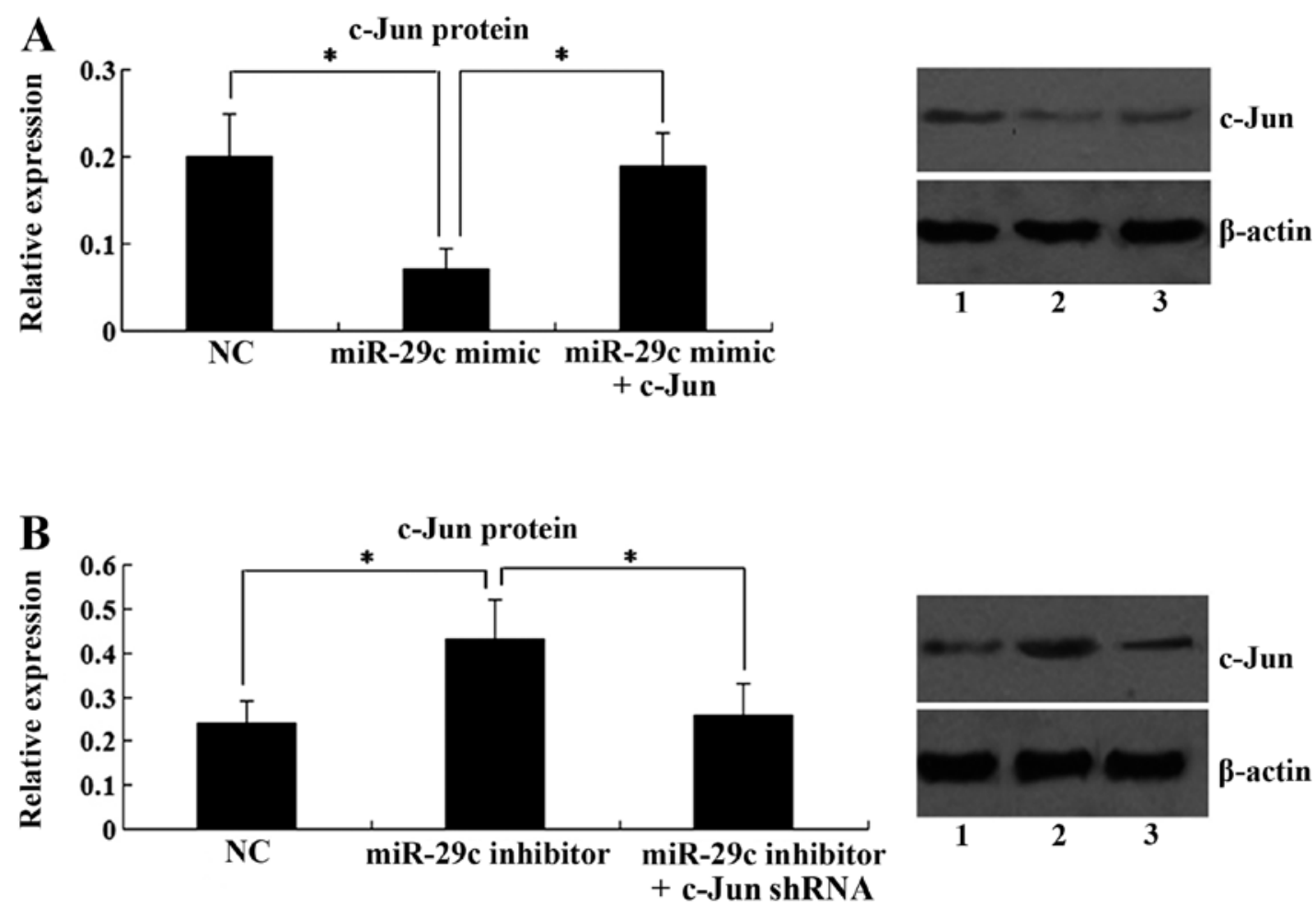

Figure 6. Protein expression of c-Jun following transfection with the miR-29c mimic/inhibitor and c-Jun overexpression/shRNA plasmid in CRL-7566 cells (A) c-Jun overexpression plasmid reversed the decrease in c-Jun protein expression in miR-29c mimic-tansfected cells. Lane 1, NC; lane 2, miR-29c mimic; lane 3, miR-29c mimic + c-Jun. (B) c-Jun shRNA plasmid reversed the increase in c-Jun protein expression in miR-29c inhibitor-transfected cells. Lane 1, NC; lane 2, miR-29c inhibitor; lane 3, miR-29c inhibitor + c-Jun shRNA. "P<0.05. NC, negative control.
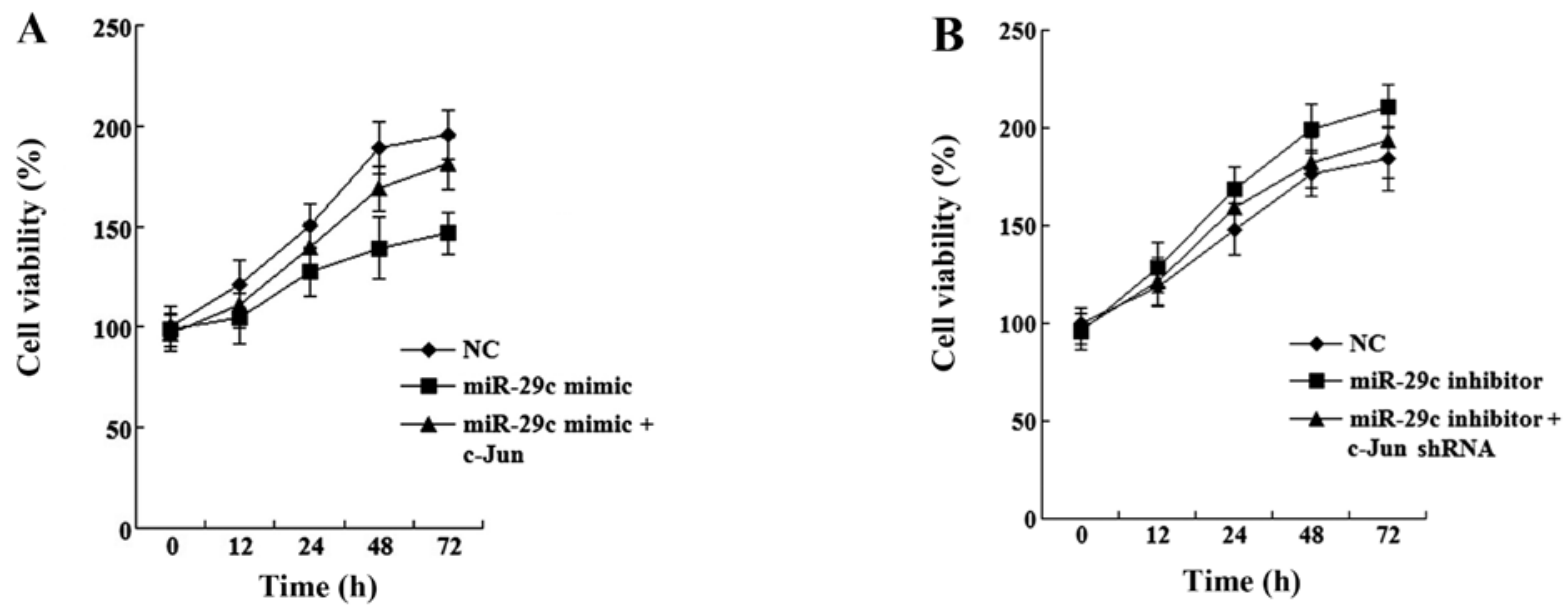

Figure 7. c-Jun reverses the miR-29c-induced suppression of cell proliferation. (A) Cell viability following transfection with the miR-29c mimic and the c-Jun overexpression vector. (B) Cell viability following transfection with the miR-29c inhibitor and the c-Jun shRNA. NC, negative control.

of the miR-29 family in the endometrium samples from women without endometriosis, as well as in paired ectopic and eutopic endometrial tissue samples from patients with endometriosis were examined by RT-qPCR. It was revealed that miR-29c was differentially expressed in the paired eutopic and ectopic endometrium samples. These findings indicate that miR-29c may be associsated with the progression of endometriosis.

To determine the potential role of miR-29c in endometrial cells, miR-29c mimic and miR-29c inhibitor were transfected into CRL-7566 cells. MTT assay revealed that the cells trans- fected with the miR-29c mimic proliferated at a significantly lower rate, and transfection with miR-29c inhibitor had the opposite effect. Endometriotic cells have an invasive potential which may contribute to the pathogenesis of endometriosis (10). In the present study, we found that transfection with miR-29c mimic suppressed cell invasion ability, while transfection with the miR-29c inhibitor enhanced cell invasion ability. FCM was used to examine the effects of miR-29c on cell apoptosis. As expected, transfection with miR-29c mimic increased the cell apoptotic rate, while transfection with the miR-29c inhibitor 


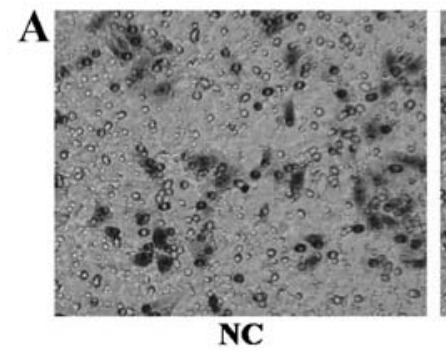

NC

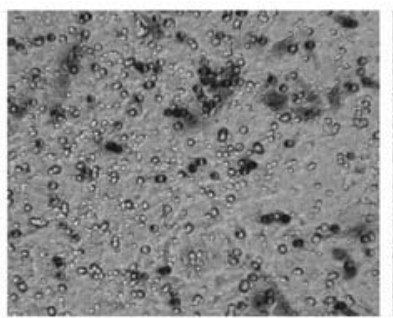

miR-29c mimic

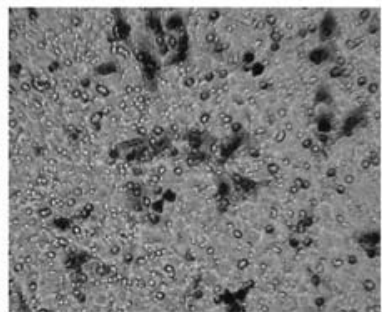

miR-29c mimictc-Jun

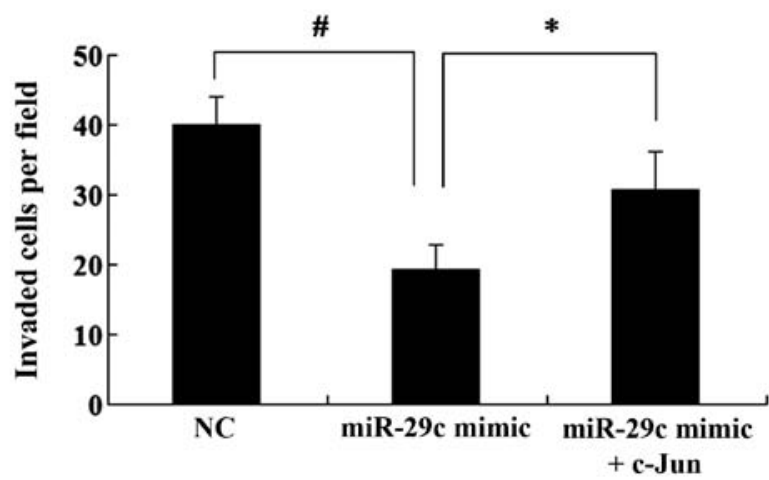

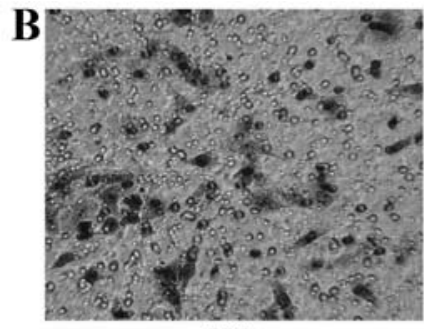

NC

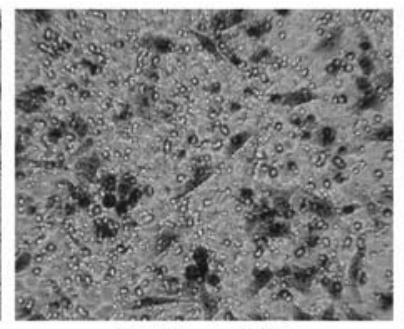

miR-29c inhibitor

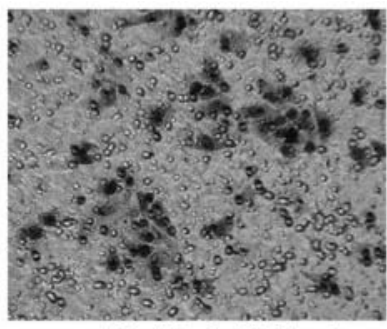

miR-29c inhibitort

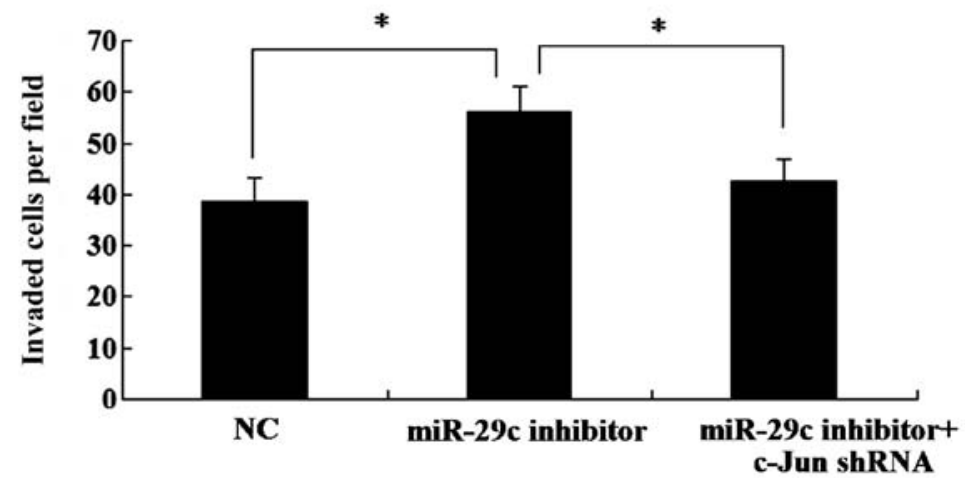

Figure 8. c-Jun reverses the miR-29c-induced suppression of cell invasion. (A) Number of invaded cells following transfection with the miR-29c mimic and the c-Jun overexpression vector. (B) Number of invaded cells following transfection with the miR-29c inhibitor and the shRNA-c-Jun. ${ }^{*} \mathrm{P}<0.05$ and ${ }^{\sharp} \mathrm{P}<0.01$. NC, negative control.
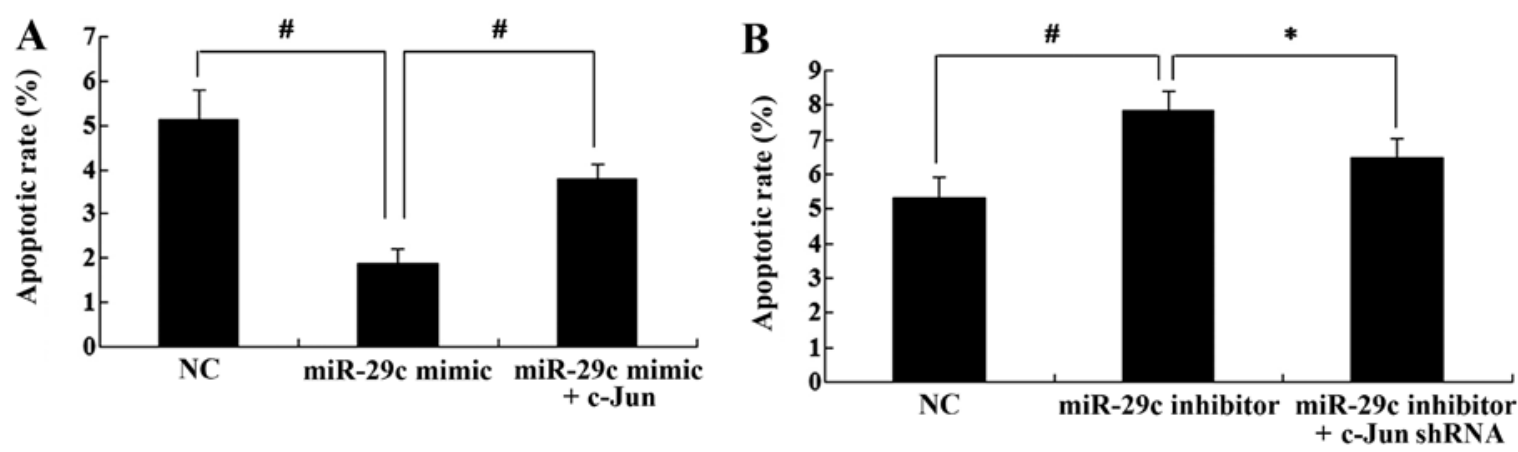

Figure 9. c-Jun reverses the miR-29c-mediated induction of cell apoptosis. (A) Cell apoptotic rate following transfection with the miR-29c mimic and the c-Jun overexpression vector. (B) Cell apoptotic rate following transfection with the miR-29c inhibitor and the shRNA-c-Jun. ${ }^{*} \mathrm{P}<0.05$ and ${ }^{*} \mathrm{P}<0.01$. NC, negative control. 
had the opposite effect. These data indicate that miR-29c potentially plays a negative role in the development of endometriotic lesions. miR-29c may exert its effects on endometrial cells by suppressing cell proliferation and invasion, as well as by promoting cell apoptosis.

Studies on the differences in gene expression between the ectopic and eutopic endometrium (17-22) have found that many genes related to cell adhesion, cell migration, cell proliferation, immune regulation and inflammation are differentially expressed in ectopic vs. eutopic endometrial tissue. In the study by Ohlsson Teague et al (12), it was suggested that c-Jun mRNA expression was significantly increased in endometriosis. c-Jun is a transcription factor which belongs to the activating protein 1 family (23). Genetic studies have demonstrated that c-Jun regulates a variety of genes important for diverse cellular functions, including cell differentiation, apoptosis, proliferation, migration and invasion (24-26). The overexpression of c-Jun has been detected in various types of tumors $(27,28)$. In the present study, it was revealed that c-Jun was also differentially expressed in the ectopic vs. eutopic endometrial tissue as determined by western blot analysis. This result is consistent with the results of a previous study demonstrating that c-Jun is associated with endometriosis (12).

We predicted the targets of miR-29c by miRanda and focused on c-Jun. Luciferase reporter assay and western blot analysis revealed that miR-29c directly binds to the c-Jun gene and inhibits the protein expression of c-Jun. These results indicate a negative role of miR-29c in the regulation of c-Jun expression and provide support for our hypothesis that miR-29c suppresses the progression of endometriosis possibly through the downregulation of c-Jun. In addition, c-Jun overexpression plasmid and shRNA plasmid were transfected into the CRL-7566 cells. The results revealed that the overexpression of c-Jun reversed the inhibitory effects of the miR-29c mimic on cell proliferation and invasion, and the overexpression of c-Jun reversed the increase in the cell apoptotic rate induced by the miR-29c mimic. In addition, c-Jun shRNA reversed the effects of the miR-29c inhibitor on cell proliferation, invasion and apoptosis. Thus, our results suggest that c-Jun plays an important role in the regulation of the proliferation, invasion and apoptosis of endometrial cells by miR-29c.

In conclusion, we identified miR-29c as a suppressor of endometriosis, and that it is differentially expressed in paired ectopic and eutopic endometrial tissues. miR-29c exerts its effects on endometrial cell proliferation, apoptosis and invasion by inhibiting the expression of c-Jun. This study may provide a novel potential therapeutic target for the treatment of endometriosis.

\section{References}

1. Giudice LC: Clinical practice. Endometriosis. N Engl J Med 362: 2389-2398, 2010.

2. Painter JN, Anderson CA, Nyholt DR, et al: Genome-wide association study identifies a locus at $7 \mathrm{p} 15.2$ associated with endometriosis. Nat Genet 43: 51-54, 2011.

3. Simpson JL, Elias S, Malinak LR and Buttram VC Jr: Heritable aspects of endometriosis. I. Genetic studies. Am J Obstet Gynecol 137: 327-331, 1980.

4. Anger DL and Foster WG: The link between environmental toxicant exposure and endometriosis. Front Biosci 13: 1578-1593, 2008.
5. Corwin EJ: Endometriosis: pathophysiology, diagnosis, and treatment. Nurse Pract 22: 35-8, 40-42, 45-46, passim; quiz 56-57, 1997.

6. Miska EA: How microRNAs control cell division, differentiation and death. Curr Opin Genet Dev 15: 563-568, 2005.

7. Jannot $\mathrm{G}$ and Simard MJ: Tumour-related microRNAs functions in Caenorhabditis elegans. Oncogene 25: 6197-6201, 2006.

8. Vandenboom Ii TG, Li Y, Philip PA and Sarkar FH: MicroRNA and cancer: tiny molecules with major implications. Curr Genomics 9: 97-109, 2008.

9. Segura MF, Hanniford D, Menendez S, et al: Aberrant miR-182 expression promotes melanoma metastasis by repressing FOXO3 and microphthalmia-associated transcription factor. Proc Natl Acad Sci USA 106: 1814-1819, 2009.

10. Gaetje R, Kotzian S, Herrmann G, Baumann R and StarzinskiPowitz A: Invasiveness of endometriotic cells in vitro. Lancet 346: 1463-1464, 1995.

11. Pan Q, Luo X, Toloubeydokhti T and Chegini N: The expression profile of micro-RNA in endometrium and endometriosis and the influence of ovarian steroids on their expression. Mol Hum Reprod 13: 797-806, 2007.

12. Ohlsson Teague EM, Van der Hoek KH, Van der Hoek MB, et al: MicroRNA-regulated pathways associated with endometriosis. Mol Endocrinol 23: 265-275, 2009.

13. Gong J, Li J, Wang Y, et al: Characterization of microRNA-29 family expression and investigation of their mechanistic roles in gastric cancer. Carcinogenesis 35: 497-506, 2014.

14. Fan YC, Mei PJ, Chen C, Miao FA, Zhang H and Li ZL: MiR-29c inhibits glioma cell proliferation, migration, invasion and angiogenesis. J Neurooncol 115: 179-188, 2013.

15. Nygren MK, Tekle C, Ingebrigtsen VA, et al: Identifying microRNAs regulating B7-H3 in breast cancer: the clinical impact of microRNA-29c. Br J Cancer 110: 2072-2080, 2014.

16. Bae HJ, Noh JH, Kim JK, et al: MicroRNA-29c functions as a tumor suppressor by direct targeting oncogenic SIRT1 in hepatocellular carcinoma. Oncogene 33: 2557-2567, 2014

17. Meola J, Rosa e Silva JC, Dentillo DB, et al: Differentially expressed genes in eutopic and ectopic endometrium of women with endometriosis. Fertil Steril 93: 1750-1773, 2010.

18. Honda H, Barrueto FF, Gogusev J, Im DD and Morin PJ: Serial analysis of gene expression reveals differential expression between endometriosis and normal endometrium. Possible roles for AXL and $\mathrm{SHCl}$ in the pathogenesis of endometriosis. Reprod Biol Endocrinol 6: 59, 2008.

19. Hu WP, Tay SK and Zhao Y: Endometriosis-specific genes identified by real-time reverse transcription-polymerase chain reaction expression profiling of endometriosis versus autologous uterine endometrium. J Clin Endocrinol Metab 91: 228-238, 2006.

20. Wu Y, Kajdacsy-Balla A, Strawn E, et al: Transcriptional characterizations of differences between eutopic and ectopic endometrium. Endocrinology 147: 232-246, 2006.

21. Eyster KM, Klinkova O, Kennedy V and Hansen KA: Whole genome deoxyribonucleic acid microarray analysis of gene expression in ectopic versus eutopic endometrium. Fertil Steril 88: 1505-1533, 2007.

22. Hull ML, Escareno CR, Godsland JM, et al: Endometrialperitoneal interactions during endometriotic lesion establishment. Am J Pathol 173: 700-715, 2008.

23. Karin M, Liu Z and Zandi E: AP-1 function and regulation. Curr Opin Cell Biol 9: 240-246, 1997.

24. Shaulian E and Karin M: AP-1 as a regulator of cell life and death. Nat Cell Biol 4: E131-E136, 2002.

25. Eferl R and Wagner EF: AP-1: a double-edged sword in tumorigenesis. Nat Rev Cancer 3: 859-868, 2003.

26. Karin M and Gallagher E: From JNK to pay dirt: jun kinases, their biochemistry, physiology and clinical importance. IUBMB Life 57: 283-295, 2005.

27. Okutomi Y, Shino Y, Komoda F, et al: Survival regulation in pancreatic cancer cells by c-Jun. Int J Oncol 23: 1127-1134, 2003.

28. Neyns B, Katesuwanasing, Vermeij J, et al: Expression of the jun family of genes in human ovarian cancer and normal ovarian surface epithelium. Oncogene 12: 1247-1257, 1996. 